\title{
Folate, genomic stability and colon cancer: the use of single cell gel electrophoresis in assessing
} the impact of folate in vitro, in vivo and in human biomonitoring.

\author{
CATALA, G.N., BESTWICK, C.S., RUSSELL, W.R., TORTORA, K., \\ GIOVANNELLI, L., MOYER, M.P., LENDOIRO, E., DUTHIE, S.J.
}




\section{Accepted Manuscript}

Title: Folate, genomic stability and colon cancer: the use of single cell gel electrophoresis in assessing the impact of folate in vitro, in vivo and in human biomonitoring

Authors: Gema Nadal Catala, Charles S Bestwick, Wendy R Russell, Katia Tortora, Lisa Giovannelli, Mary Pat Moyer, Elena Lendoiro, Susan J. Duthie

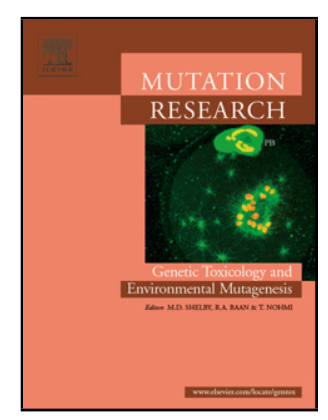

PII:

DOI:

Reference:

S1383-5718(18)30264-X https://doi.org/10.1016/j.mrgentox.2018.08.012

To appear in: MUTGEN 402961

Received date:

Revised date:

Mutation Research

Accepted date:

$17-7-2018$

29-8-2018

29-8-2018

Please cite this article as: Catala GN, Bestwick CS, Russell WR, Tortora K, Giovannelli L, Moyer MP, Lendoiro E, Duthie SJ, Folate, genomic stability and colon cancer: the use of single cell gel electrophoresis in assessing the impact of folate in vitro, in vivo and in human biomonitoring, Mutation Research - Genetic Toxicology and Environmental Mutagenesis (2018), https://doi.org/10.1016/j.mrgentox.2018.08.012

This is a PDF file of an unedited manuscript that has been accepted for publication. As a service to our customers we are providing this early version of the manuscript. The manuscript will undergo copyediting, typesetting, and review of the resulting proof before it is published in its final form. Please note that during the production process errors may be discovered which could affect the content, and all legal disclaimers that apply to the journal pertain. 
Folate, genomic stability and colon cancer: the use of single cell gel electrophoresis in assessing the impact of folate in vitro, in vivo and in human biomonitoring.

Running Title: The comet assay for measuring the impact of folate status on genomic instability and DNA methylation

Gema Nadal Catala ${ }^{1}$, Charles S Bestwick ${ }^{1}$, Wendy R Russell ${ }^{1}$, Katia Tortora ${ }^{2}$, Lisa Giovannelli², Mary Pat Moyer ${ }^{3}$, Elena Lendoiro ${ }^{4,5}$ and Susan J. Duthie ${ }^{1,5^{*}}$

${ }^{1}$ Natural Products Group, Division of Lifelong Health, Rowett Institute of Nutrition and Health, University of Aberdeen, Aberdeen, UK.

2 Department NEUROFARBA, Section of Pharmacology and Toxicology, University of Florence, Florence, Italy.

${ }^{3}$ INCELL Corporation, San Antonio, Texas, USA

${ }^{4}$ Department of Toxicology, Institute of Forensic Sciences, University of Santiago of Compostela, Santiago de Compostela, Spain.

${ }^{5}$ School of Pharmacy and Life Sciences, Robert Gordon University, Aberdeen, UK.

*Professor Susan J. Duthie, Robert Gordon University, School of Pharmacy and Life Sciences, Garthdee Road, Aberdeen, AB10 7GJ, UK

Tel +44 1224262815

E-mail s.j.duthie@rgu.ac.uk 
Highlights from this short review include:

- the comet assay in assessing genomic stability in relation to folate status and human cancer

- impact of folate deficiency/supplementation on genomic stability in vitro, in rodents and humans

- the impact of MTHFR genotype on folate status and genomic stability in human carcinogenesis

- adaptation of the comet assay in measuring DNA methylation and impact on diet and epigenetics

\begin{abstract}
Intake of folate (vitamin $\mathrm{B}_{9}$ ) is strongly inversely linked with human cancer risk, particularly colon cancer. In general, people with the highest dietary intake of folate or with high blood folate levels are at a reduced risk (approx. 25\%) of developing colon cancer.

Folate acts in normal cellular metabolism to maintain genomic stability through the provision of nucleotides for DNA replication and DNA repair and by regulating DNA methylation and gene expression. Folate deficiency can accelerate carcinogenesis by inducing misincorporation of uracil into DNA, by increasing DNA strand breakage, by inhibiting DNA base excision repair capacity and by inducing DNA hypomethylation and consequently aberrant gene and protein expression. Conversely, increasing folate intake may improve genomic stability.
\end{abstract}

This review describes key applications of single cell gel electrophoresis (the comet assay) in assessing genomic instability (misincorporated uracil, DNA single strand breakage and DNA repair capacity) in response to folate status (deficient or supplemented) in human cells in vitro, in rodent models and in human case-control and intervention studies. It highlights an adaptation of the SCGE comet assay for measuring genome-wide and gene-specific DNA methylation in human cells and colon tissue.

Keywords: single cell gel electrophoresis, comet assay, folate, genomic stability, colon, uracil, DNA methylation 


\section{Introduction}

Single cell gel electrophoresis (SCGE) or the comet assay is now established in human biomonitoring as a sensitive and specific assay capable of measuring low levels of DNA strand breakage, altered DNA bases and DNA repair capacity in human populations. However, the comet assay is also a particularly valuable investigative tool for exploring experimental mechanisms of genotoxicity linked to genomic instability, malignant transformation and carcinogenesis.

Intake of vitamin B9 (folate) is strongly linked with human cancer risk [1-4]. This review describes several important modifications of the comet assay for assessing DNA instability in response to folate status in human cells in vitro, in rats in vivo and in human studies, and highlights an emerging application of the SCGE assay in measuring aberrant DNA methylation.

\section{Folate status and human cancers}

Folates, a family of water-soluble B vitamins are found in a wide variety of foods including green leafy vegetables, cereals, beans, fruit and liver, and play a crucial role in preventing the development of human pathologies such as cancer and heart disease in adults, cognitive dysfunction and dementia in the elderly and congenital defects in babies. In the UK, suboptimal folate status is common, particularly in adolescents and the elderly. Overt folate deficiency has been implicated in the development of several human malignancies including cancer of the breast, ovary, lung and cervix [1,2]. Moreover, the evidence linking low folate status with an increased risk of colon cancer (CC) is particularly strong [1,2]. Globally, CC is the $3^{\text {rd }}$ most commonly diagnosed cancer in the world (http://globocan.iarc.fr). Recent metaanalyses of case-control and prospective studies consistently report a reduced relative risk (RR) for CC in people with a high dietary folate intake compared with people with a low intake: $\mathrm{RR} 0.75 ; 95 \% \mathrm{Cl}=0.64-0.89$ [3]; $\mathrm{RR} 0.81 ; 95 \% \mathrm{Cl}=0.66-0.99$ [4].

\section{Folate and mechanisms of carcinogenesis}

Folate is critical in one-carbon metabolism and acts to maintain genomic stability by regulating DNA biosynthesis and DNA repair and by controlling DNA methylation and gene expression (Figure 1). Within the methionine cycle, 5-methyltetrahydrofolate (5-methylTHF) 
remethylates homocysteine to methionine, which is further metabolised to $\mathrm{S}$ adenosylmethionine (SAM). SAM controls gene transcription and ultimately protein expression through its ability to methylate cytosine in the DNA molecule. Similarly, folate is essential for the synthesis of both purines and the pyrimidine nucleoside thymidine. Deoxyuridine monophosphate (dUMP) is converted to deoxythymidine monophosphate (dTMP) by thymidylate synthase (TS), using 5,10-methylenetetrahydrofolate $(5,10$ methyleneTHF) as methyl donor. Subsequently, 5,10-formyltetrahydrofolate $(5,10$ formylTHF) is involved in the production of both adenosine and guanosine. Continual production of these DNA precursors is essential for normal DNA synthesis and for effective DNA repair. Folate insufficiency induces and accelerates carcinogenesis by perturbing each of these processes [1,2]. If dietary folate is limiting, the balance of purine and pyrimidine DNA precursors is altered and normal DNA synthesis and DNA repair are inhibited, leading to mutagenesis and malignant transformation. Moreover, folate depletion blocks the normal methylation of dUMP to dTMP, ultimately causing an accumulation of deoxyuridine triphosphate (dUTP). As dUTP and deoxythymidine triphosphate (dTTP) differ only by a single methyl group, the RNA base uracil, which is not normally present in DNA, is misincorporated into the DNA molecule in place of thymine (Figure 2). This mismatch quickly triggers DNA base excision repair (BER) processes that remove the uracil from the DNA. However, if folate availability continually limits the synthesis of thymine, then uracil is misincorporated and cleaved from the DNA in what is described as "a catastrophic repair cycle", resulting in DNA double strand breakage, chromosomal damage and ultimately malignant transformation [5]. Folate deficiency similarly perturbs the production of S-adenosylmethionine (SAM; Figure 3), causing a reduction in the normal methylation of cytosine in the DNA molecule and leading to global DNA hypomethylation and inappropriate proto-oncogene activation $[6,7]$. This will be addressed in detail later in this review.

\section{Using SCGE to understand the impact of folate status on genomic stability}

\subsection{Misincorporated uracil}

As described above, folate deficiency induces misincorporation of uracil into DNA, subsequently inducing DNA single and double strand breakage and ultimately chromosomal 
damage and malignant transformation [1,5]. A valuable modification of the alkaline comet assay has allowed for uracil to be detected and measured specifically in human and animal cells and tissues. Here, DNA nucleoids are lysed and incubated with the bacterial DNA repair enzyme uracil DNA glycosylase (UDG; 1 unit/ $\mu \mathrm{g}$ DNA for $30 \mathrm{~min}$ ) which removes misincorporated uracil from the DNA molecule, leaving a single strand break which is detected by the comet assay in the standard manner [8]. This assay, specifically developed to measure uracil misincorporation, has been employed in the study of human peripheral blood mononuclear cells (PBMC), epithelial cells and colonocytes in vitro [8-10], in rodent studies $[11,12]$ and in both human observational and intervention studies $[13,14]$ to determine the influence of folate status on DNA stability.

Growing human cells in folate-free medium inhibits DNA synthesis and progressively increases uracil misincorporation (2-3-fold) in PBMC ex vivo [9], in human colonocytes [15], and in SV40-immortalised human colon epithelial cells (HCEC) with time in culture [10]. This detrimental effect of folate deficiency on DNA stability in HCEC was highly sensitive to folate, with uracil concentrations declining to baseline levels after repleting the cells with folate at concentrations normally observed in both human blood and colon tissue (10 ng/mL; [10]).

Nutritional folate deficiency has also been shown to induce DNA instability in rodents in vivo. Here, DNA uracil misincorporation and DNA single strand breakage increased 2-fold $(P<0.001)$ in PBMC isolated from male Rowett Hooded Lister rats fed a folate-free diet either for 10 or 24 weeks [11,12]. However, the modified comet assay is probably most valuable when deployed in biomonitoring to understand better the relationship between folate and DNA stability in humans.

While elevated uracil misincorporation has been measured [by gas chromatography mass spectrometry (GC MS) or the highly invasive deoxurydine assay] in PBMC and bone marrow from patients with severe folate deficiency and/or megaloblastic anaemia [5,16], little work has been carried out to establish whether there may be a beneficial effect on DNA stability by improving folate status in the general population. Supplementing apparently healthy men $(n=15)$ and women $(N=15)$ (mean age 41 years old with a red blood cell folate level of 250$650 \mathrm{nmol} / \mathrm{L})$ with folic acid (1.2 mg/day) for 12 weeks substantially increased red blood cell folate (approx. 90\%; $\mathrm{P}<0.01$ ), PBMC folate (approx. 85\%; $\mathrm{P}<0.01$ ) and plasma 5-methylTHF (approx. 300\%; $P<0.01$ ), when compared with participants taking placebo [17]. Low levels of uracil were detected using SCGE in the PBMC of these healthy participants, and this strongly 
correlated with PBMC folate (Figure 4; $r=-0.49, \mathrm{P}<0.01$ ), plasma 5-methylTHF $(r=-0.48$, $\mathrm{P}<0.01)$ and intracellular red cell folate status $(r=-0.46, P<0.01)$ both pre- and postintervention. Moreover, increasing blood folate concentration decreased significantly the level of uracil measured in PBMC isolated from subjects in the intervention group (approx. $20 \%, P<0.05)$, highlighting that genomic stability can be improved by increasing folate levels even in folate-sufficient subjects $[14,17]$. However, the majority of human biomonitoring studies are (necessarily) limited in that they measure genomic stability in blood cells rather than in the target organ. Nonetheless, a few studies have determined the direct impact of folate status on genomic stability and specifically uracil misincorporation in the colon. In a study of patients with adenomatous $(n=40)$ or hyperplastic polyps $(n=16)$, folate levels in colon biopsy samples decreased (as much as $25 \%$ ) with proximity to the polyp site when compared with control subjects $(n=53)$. Moreover, there was a corresponding increase in misincorporated uracil (measured using the UDG-modified comet assay) [18]. In a follow-up intervention study by the same research group [19], uracil misincorporation ratio (UDGmodified comet assay) declined by $0.5(\mathrm{P}<0.05)$ at the site closest to the original polyp in polypectomised patients following treatment with folic acid (600 $\mu \mathrm{g} /$ day) for six months.

These findings show that uracil misincorporation is responsive to folate status both in surrogate and target tissue.

\subsection{DNA strand breakage, DNA repair capacity and apoptosis}

There are currently two ways in which the comet assay is used to measure DNA BER; the cellular repair assay, where whole cells are exposed in culture to a model damaging agent and complete repair of DNA strand breaks or DNA lesions is followed with time; and the in vitro cell-free repair assay where repair enzymes in a prepared cell extract (normally PBMC) incise model substrate DNA that contains an induced and specific lesion. Both of these assays have been used to assess how folate status affects DNA BER activity.

DNA BER capacity (cellular assay) is inhibited in folate-depleted human PBMC ex vivo and in SV40-immortalised HCEC in response to both oxidative (hydrogen peroxide) and alkylation (methyl-methane-sulfonate) damage $[9,10]$.

A similar effect has been observed in non-transfected normal human colon epithelial cells. Originally isolated from a 68 year old Hispanic male, NCM460 cells (obtained under a material 
transfer agreement from INCELL, San Antonio USA) have been used to model B vitamin uptake and metabolism in the human intestine [20] and to assess the effect of folate deficiency on genomic stability [15]. The experiment described below demonstrates how folate depletion alters endogenous DNA strand breakage, DNA BER activity (measured using the comet assay), and ultimately apoptosis, in non-tumour derived human colon cells.

NCM460 cells were grown in A52 medium (BioConcept, Switzerland) in the absence (F-) or presence of folic acid ( $\mathrm{F}+; 4 \mu \mathrm{g} / \mathrm{mL}$ ). Intracellular total folate (measured by radioassay; [15]), cell proliferation (cell number), endogenous DNA strand breakage (comet assay), DNA BER capacity (comet assay) and apoptosis [polycaspase $(1,3,4-9)$ activity using Vibrant FAM polycaspase assay kit (Invitrogen, UK) and by Flow cytometry (4 colour FACS Calibur, Becton Dickinson, Oxford, UK)] were measured up to 15 days in culture.

Intracellular total folate was depleted by more than $90 \%$ in NCM460 cells cultured in the absence of folic acid while cell growth was progressively inhibited to approx. $33 \%$ of the levels observed in folate supplemented cultures after 15 days (data not shown). Folate depletion induced genomic instability in normal human colonocytes, observed as a progressive increase in endogenous DNA strand breakage (Figure 5) and inhibition of DNA BER capacity (Figure 6) in response to oxidative challenge $\left(20 \mu \mathrm{M} \mathrm{H}_{2} \mathrm{O}_{2}\right)$. In this experiment, DNA strand breaks increased more than 2-fold [69.3 compared with $32.1 \mathrm{AU}$ in F-versus F+ NCM460 cells respectively $(P<0.01)]$ and $B E R$ rate was approx. $17 \%$ lower in folate depleted NCM460 after 14 days [3.67 compared with $4.43 \mathrm{AU} / \mathrm{min}$ respectively $(\mathrm{P}<0.05)$ ]. Additionally, this timedependent decrease in genomic stability as a consequence of folate depletion, translated into increased programmed cell death.

Caspase activation is used as an initial marker for the induction of programmed cell death such as results from folate depletion. Caspases are a family of cysteine proteases linked to programmed cell death events, and in particular to the development and execution of apoptosis [21]. Apoptosis is intimately linked to maintaining genomic stability by removal of damaged cells, thereby reducing the risk of malignant transformation leading to tumorigenesis.

While folate deficiency was initially associated with a small decrease in polycaspase activation as detected by flow cytometric analysis using the vibrant FAM polycaspase assay (which identifies the majority of caspase activies including caspase-1, 2, 4, 5, 7 and 9) in human colonocytes, this was followed by a substantial increase (approx. $50 \%$ ) in enzyme activity after 
14 days in culture (Figure 7A). This elevation was observed without any concomitant change in colonocyte cell membrane integrity (as measured by propidium iodide uptake), indicating the likelihood of an apoptotic rather than necrotic mechanism of cell death (Figure 7B). These data confirm that folate deficiency detrimentally impacts on genomic stability in normal human colon epithelial cells, by inducing DNA strand breakage and concurrently inhibiting effective DNA repair, resulting in an increased activity linked to elimination of critically damaged colonocytes from the population.

We have also shown that BER capacity is sensitive to folate status in humans. In the human intervention study described above, BER repair capacity in participants receiving a folic acid supplement (1.2 mg daily for 12 weeks) or control participants was measured by employing the cell-free DNA BER comet assay. Extracts were prepared from PBMC sampled from the participants at the start and at the end of the study. Excision repair capacity was measured as the ability of PBMC extracts to excise 8-oxo-7,8-dihyroguanine (8-oxodG) from Chinese hamster Ovary (CHO) DNA over 20 min following exposure to the model sensitiser RO19-8022 [14]. Excision of 8-oxodG leaves a strand break, which is measured using the conventional alkaline comet assay. Increasing folate concntration in the majority of these normal healthy study participants had no significant effect on BER capacity. However, folate supplementation significantly reduced incision repair activity in those subjects with the lowest initial baseline red cell folate levels suggesting either that increased provision of DNA nucleotides or a reduction in DNA damage, may downregulate BER capacity only in relatively folate-depleted individuals [14].

\section{The impact of nutrient-gene interactions on folate metabolism and genomic stability}

An important but relatively neglected area of investigation pertaining to folate status and cancer risk is the impact of nutrient:gene interactions on genomic stability. The enzyme methylenetetrahydrofolate reductase (MTHFR; EC 1.5.1.20), which irreversibly converts 5,10methyleneTHF to 5-methylTHF (Figure 1), is a pivotal protein in folate metabolism which,

when functional, directs methyl groups away from the production of thymidine towards homocysteine remethylation and the production of SAM. Two human polymorphisms in MTHFR (C677T and A1298C) are associated with a reduced enzyme activity, low circulating folate, low SAM and elevated homocysteine levels. Given that low SAM would result in an 
increased likelihood of DNA hypomethylation, and contrary to what might be expected, these variants are actually associated with a reduced risk of CC [22]. One hypothesis is that the homozygous recessive variant reduces genomic instability by ensuring the continual production of nucleotides for DNA synthesis and repair (Figure 1). In a study of 199 individuals without cancer, homozygosity for the C677T polymorphism was associated with a reduced plasma and red cell folate (both approx. 25\%) level, and decreased hyper-homocysteinemia (approx. 21\%) when compared with wild type individuals. However, DNA strand breakage and uracil misincorporation (both measured by SCGE) were similar for all genotypes, indicating that in this study sequestration of 5,10-methyleneTHF for thymidine synthesis was not a major contributor to the apparent enhanced genomic stability in C677TT homozygous variants [13]. Nonetheless, other groups have found a lower level of plasma folate and of misincorporated uracil (measured in leukocytes using the uracil DNA glycosylase-modified comet assay) in individuals carrying the C677T polymorphism [23]. However, this study is limited by both the small sample size $(n=14)$ and the need to combine data for the C677CT and 677TT genotypes for the analyses.

\section{Innovations in the comet assay to detect DNA methylation}

While the comet assay is now used widely to investigate the influence of folate on DNA repair capacity and genomic stability, new variations are being developed to improve further the specificity and effectiveness of the assay in establishing folate-modulated mechanisms of carcinogenesis.

It is now established that epigenetic changes, including aberrant DNA methylation, play a critical role in human carcinogenesis [24]. Moreover, it is estimated that dysregulated DNA methylation is involved in the aetiology of approx. one fifth of all CC cancer cases [25].

Folate (5-methylTHF) plays a critical role in the normal regulation of gene and protein expression by acting as methyl donor for the methylation of cytosines in the DNA molecule. Folate deficiency disrupts normal DNA methylation by attenuating the remethylation of SAH to SAM and inducing DNA hypomethylation in newly synthesised DNA. This effectively allows for the inappropriate expression (or over-expression) of genes, including proto-oncogenes, that increase the risk of malignant transformation and cancer [7; Figure 3]. 
Methods for measuring genomic DNA methylation, which include Southern blotting, liquid chromatography tandem mass spectrometry (LCMSMS/MS) and a nucleotide extension assay, are expensive, require considerable biological material and can provide inconsistent data. As aberrant DNA methylation plays a role in both the early development of CC (global hypomethylation) and in advanced stages of tumours (gene-specific hypermethylation), the ability to detect and quantify aberrant methylation from blood samples or small tumour biopsies would offer a valuable predictive and prognostic biomarker. A methyl-sensitive variation of the SCGE assay has been described previously. This assay compared with other methods is simpler and more cost-effective [26-29]. For this modification, two isoschizomer endonucleases (Hpall and Mspl) that share the same restriction site in CpG islands are deployed separately in the assay at the post-lysis step. As only Hpall activity is blocked by methylation in the restriction site, the level of Hpall digestion is inversely proportional to the level of global DNA methylation. Thus, global methylation changes can be evaluated as the ratio between the comet scores after the two separate enzymic digestions.

We have developed this method further and tested its ability to detect perturbations in DNA methylation in human colon cells as a consequence of folate depletion (hypomethylation) or exposure to nickel chloride which has been shown to induce DNA hypermethylation [28]. To induce a state of DNA hypomethylation, NCM460 human colon epithelial cells (cultured in A52 medium as described previously) or primary human colon fibroblasts [CCD-18Co obtained from the American Type Culture Collection and cultured in DMEM with low glucose $(1.0 \mathrm{~g} / \mathrm{L})$ ] were grown for $48 \mathrm{~h}$, or 7 days, either in regular culture medium containing folic acid $(4 \mu \mathrm{g} / \mathrm{mL})$ or in the same medium without folic acid (BioConcept Inc, Switzerland). To induce DNA hypermethylation, colonocytes and fibroblasts in folate sufficient medium were exposed to $\mathrm{NiCl}_{2}(500 \mu \mathrm{M})$ for $24 \mathrm{~h}$. SCGE was carried out essentially as described previously with the subsequent addition of a fast digest endonuclease enzyme incubation step [27]. Post lysis, the slides were washed three times for 5 min each with PBS $\left(4^{\circ} \mathrm{C}\right)$ before incubation either with Hpall, Mspl or Fast Digest Buffer [FDB; (ThermoFisher Scientific Inc, Massachusetts, USA] in a humidified chamber at $37^{\circ} \mathrm{C}$ for $15 \mathrm{~min}$. At the end of this incubation, the slides were subjected to alkaline unwinding (40 min), electrophoresis (40 min), staining (DAPI $1 \mu \mathrm{g} / \mathrm{mL}$ ) and visual scoring as normal [30].

Genome-wide DNA methylation was calculated as the ratio between the two separate enzyme digestions after accounting for the effect of buffer alone [28], with a higher ratio 
reflecting a lower level of DNA methylation. $\mathrm{NiCl}_{2}$ induced DNA hypermethylation in both human colon cell lines (Figure 8A). However, the effect was statistically significant only in CCD18Co fibroblasts. Short-term folate depletion (48h) increased the Hpall/Mspl ratio approx. 3fold for the epithelial and 4-fold for the colon fibroblast cells (Figure 8B), indicating that Hpall digestion was, as expected, more effective, when DNA methylation is lower. A similar result was observed after 7 days in response to longer-term folate deficiency (Figure 8B).

These data indicate that this modification of the comet assay to detect DNA methylation in single cells is responsive to known modifiers of methylation status. Similar results have been reported using another methylation-sensitive variant of the comet assay [31]. Here DNA methylation status was measured by SCGE in folate deficient or replete human colonic adenocarcinoma SW620 cells (up to 14 days) using the methylation-sensitive restriction enzymes Hpall and Hhal (2.5U/gel for 1h). DNA global hypomethylation increased significantly (approx.6-fold) in folate depleted cells. Moreover, there was also significant DNA hypomethylation detected in the p53 gene region in these cells (approx. 2-3 fold), measured using a particularly elegant variant of the methylation-sensitive comet assay, which combines SCGE and FISH [31].

In view of the role of aberrant DNA methylation in cancer development, the ability to adapt the comet assay to specifically detect changes in DNA methylation patterns in human tissue will undoubtedly advance understanding of how epigenetics influences human cancer risk. An initial application of a methylation-sensitive variant of the comet assay [31] in the context of human carcinogenesis demonstrated that folate levels are significantly lower in colon tissue biopsies from patients with pre-cancerous polyps compared with non-diseased controls, and this is associated with genome-wide DNA hypomethylation [18]. Moreover, both colonocyte folate and DNA methylation declined with proximity to the original polyp site [18]. Global aberrant DNA methylation was found to be responsive to folate supplementation in a subsequent intervention study, which demonstrated that taking folic acid $(600 \mu \mathrm{g} / \mathrm{day}$ for 6 months) decreased genome-wide DNA hypomethylation in colonocytes isolated from polypectomised patients $(n=12)$ compared with non-supplemented individuals $(n=8)$ [19].

\section{Summary}


A considerable strength of SCGE is the genuine adaptability of the assay to measure multiple biomarkers of DNA stability. This review demonstrates that employing the comet assay to measure DNA strand breakage, misincorporated uracil, DNA BER capacity and now, DNA methylation in single cells, allows us to better understand in detail, how the B vitamin folate acts mechanistically on DNA stability and malignant transformation in human and rodent models. These studies serve to highlight the sensitivity of the assay to detect, and at least partially quantify, changes in DNA stability due to intervention. Here, we have been able to establish how folate deficiency works through increased uracil misincorporation, DNA strand breakage, defective BER and DNA hypomethylation to induce or accelerate genomic instability, while conversely, improved folate status reduces misincorporated uracil in patients with colon polyps and even in apparently healthy human volunteers.

\section{Conflict of interest}

All authors declare no conflict of interest.

\section{Acknowledgements}

This work was supported by Tenovus Scotland and The Scottish Government Rural and Environmental Science and Analytical Sciences Division (RESAS). The authors are grateful to the COST Action CA15132, 'hCOMET' for funding a STSM to support the development of the methylation-sensitive comet assay and to Consellería de Cultura, Educación e Ordenación Universitaria, Xunta de Galicia, for funding Dr Elena Lendoiro her postdoctoral fellowship (ED481B2016/103-0). 
Figure 1. Folate and one-carbon metabolism: regulation of DNA synthesis, DNA repair and DNA methylation.

A simplified scheme showing how dietary and cellular folates mediate (A) normal DNA synthesis, and repair and (B) DNA methylation and gene expression

Abbreviations: dihydrofolate: DHF; tetrahydrofolate: THF; 5,10-methylenetetrahydrofolate: 5,10-methyleneTHF; 5,10-formyltetrahydrofolate: $\quad$ 5,10-formylTHF; $\quad 5$ methyltetrahydrofolate: $\quad$ 5-methylTHF; $\quad$ s-adenosylmethionine: $\quad$ SAM; $\quad s-$ adenosylhomocysteine: SAH; methylenetetrahydrofolate reductase: MTHFR; deoxyuridine monophosphate: dUMP; deoxythymidine monophosphate: dTMP; thymidylate synthase: TS. Adapted with kind permission from J. Inherited \& Metabolic Disorders [1].

\section{Figure 2. Folate deficiency and uracil misincorporation}

The impact of dietary folate deficiency on uracil misincorporation and chromosomal instability. Abbreviations: dUTP: deoxyuridine triphosphate; dTTP: deoxythymidine

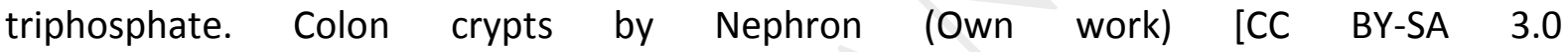
(http://creativecommons.org/licenses/by-sa/3.0) or GFDL (http://www.gnu.org/copyleft/fdl.html)] via Wikimedia Commons. Images of DNA damage are authors own.

\section{Figure 3. Folate deficiency, aberrant DNA methylation and gene expression}

The effect of dietary folate deficiency on DNA hypomethylation and proto-oncogene expression. Abbreviations: 5-methyltetrahydrofolate: 5-methylTHF; s-adenosylmethionine: SAM; s-adenosylhomocysteine: SAH; DNAMT: DNA methyltransferase. Colon crypts by Nephron (Own work) [CC BY-SA 3.0 (http://creativecommons.org/licenses/by-sa/3.0) or GFDL (http://www.gnu.org/copyleft/fdl.html)] via Wikimedia Commons.

\section{Figure 4. Association between PBMC folate status and PBMC DNA uracil misincorporation in volunteers}

Change in uracil misincorporation with increasing PBMC folate status due to intervention with folic acid (1.2mg/day for 12 weeks) in healthy human volunteers. Adapted with kind permission from the British Journal of Cancer [14]. 
Figure 5. Effect of folic acid deficiency on endogenous DNA damage

Endogenous DNA strand breakage in NCM460 cells cultured under folic acid sufficient (F+; $4 \mu \mathrm{g} / \mathrm{mL}$, black) or deficient (F-; $<1 \mathrm{ng} / \mathrm{mL}$, grey) conditions. Results are mean $\pm \operatorname{SEM}(\mathrm{n}=6)$. ${ }^{*} p<0.01$, where $\mathrm{P}$ values refer to differences between cells grown in the presence or absence of folate by Students T-test.

Figure 6. Effect of folic acid deficiency on DNA base excision repair capacity (BER) with time. NCM460 cells were grown in the presence or absence of folic acid for 7 (A) or 14 days (B) in either folic acid sufficient $(4 \mu \mathrm{g} / \mathrm{mL}, \bullet)$ or deficient $(<1 \mathrm{ng} / \mathrm{mL}, \square)$ conditions. Cells were exposed to hydrogen peroxide $(200 \mu \mathrm{g})$ and BER capacity measured over $60 \mathrm{~min}$. UT; untreated cells. Results are mean \pm SEM $(n=6)$. ${ }^{*} p<0.05$, where $P$ values refer to differences between cells grown in the presence or absence of folate by Students T-test.

Figure 7. Effect of folic acid deficiency on caspase (A) and cell membrane integrity (B) Poly-caspase activation and cell membrane permeability was measured in NCM460 cells cultured under folic acid sufficient ( $\mathrm{F}+; 4 \mu \mathrm{g} / \mathrm{ml}$, black) or deficient (F-; $<1 \mathrm{ng} / \mathrm{mL}$, grey) conditions for 7 or 14 days. Results are mean \pm SEM $(n>/=5) .{ }^{*} p<0.05,{ }^{* *} p<0.001$, where $P$ values refer to differences between cells grown in the presence or absence of folate by Students T-test.

Figure 8: Global DNA methylation measured in human colon cells and fibroblasts

The methylation-sensitive variant of the comet assay was used in NCM460 colon epithelial cells and CCD-18Co fibroblasts. (A) Effect of a hypermethylating stimulus: $500 \mu \mathrm{M} \mathrm{NiCl}$ treatment for 24h. (B) Effect of folic acid (FA) depletion for $48 \mathrm{~h}$ or 7 days [F+ $(4 \mu \mathrm{g} / \mathrm{ml})$ or F$(0 \mathrm{ng} / \mathrm{mL})]$. Results show the mean ratio between Hpall and Mspl DNA digestion $\pm S E(n=4)$. ${ }^{*} p<0.05$ where $P$ values refer to differences between treated and untreated cells by one-way ANOVA. 


\section{References}

1. S.J. Duthie, Folate and cancer: how DNA damage, repair and methylation impact on colon carcinogenesis. J Inherit. Metab. Dis. 34 (2011) 101-109.

2. Y.I. Kim, Folate and colorectal cancer: an evidence-based critical review. Mol. Nutr. Food Res. 51 (2007) 267-292.

3. M.A. Sanjoaquin, N. Allen, E. Couto, A.W. Roddam, T.J. Key, Folate intake and colorectal cancer risk: a meta-analytical approach. Int. J. Cancer 113 (2005) 825828.

4. V.L. Stevens, L M.L. McCullough, J. Sun, E.J. Jacobs, P.T. Campbell, S.M. Gapstur, High levels of folate supplements and fortification are not associated with increased risk of colorectal cancer. Gastroenterology 141 (2011) 98-105.

5. B.C. Blount, M.M. Mack, C.M. Wehr, J.T. MacGregor, R.A. Hiatt, G. Wang, S.N. Wickramasinghe, R.B. Everson, B.N. Ames, Folate deficiency causes uracil misincorporation into human DNA and chromosomal breakage; implications for cancer and neuronal damage. P.N.A.S USA 94 (1997) 3290-3295.

6. E. Wainfan, L.A. Poirier, Methyl groups in carcinogenesis: effects on DNA methylation and gene expression. Cancer Res. 52 (1992) 2071s-2077s.

7. S.J. Duthie, Epigenetic modifications and human pathologies: cancer and CVD. Proc. Nutr. Soc. 70 (2011) 47-56.

8. S.J. Duthie, P. McMillan, Uracil misincorporation in human DNA detected using single cell gel electrophoresis. Carcinogenesis 18 (1997) 1709-1714.

9. S.J. Duthie, A. Hawdon, DNA stability (strand breakage, uracil misincorporation, and defective repair) is increased by folic acid depletion in human lymphocytes in vitro. FASEB J. 12 (1998) 1491-1497.

10. S.J. Duthie, S. Narayanan, S. Blum, L. Pirie, G.M. Brand, Folate deficiency in vitro induces uracil misincorporation, DNA hypomethylation and inhibits DNA excision repair in immortalised normal human colon epithelial cells. Nutrition and Cancer 37 (2000) 127-133.

11. S.J. Duthie, G. Grant, S. Narayanan, Increased uracil misincorporation in lymphocytes from folate-deficient rats. Brit. J. Cancer 83 (2000) 1532-1537. 
12. S.J. Duthie, L.P. Pirie, G. Grant, A.J. Watson, G.P. Margison, Long term folate deficiency differentially alters hepatic and colon MGMT and OGG-1 DNA repair protein expression in rats but has no impact on genome-wide DNA methylation. Cancer Prevention Res. 3 (2010) 92-100.

13. S. Narayanan, J. McConnell, J. Little, L.Sharp, C.J. Piyathilake, H.Powers, G. Basten, S.J. Duthie. Methylenetetrahydrofolate reductase gene: Association with folate metabolism and DNA stability (strand breaks, misincorporated uracil and DNA methylation status). Cancer Epidemiol. Biomarkers Prev. 13 (2004) 1-8.

14. G.P. Basten, S.J. Duthie, L.P. Pirie, N. Vaughan, M.H. Hill, H.J. Powers. Sensitivity of markers of DNA stability and DNA repair activity to folate supplementation in healthy volunteers. Brit. J. Cancer 94 (2006) 1942-1947.

15. S.J. Duthie, Y. Mavrommatis, G. Rucklidge, M. Reid, G. Duncan, M.P. Moyer, L.P. Pirie, C.S. Bestwick. The response of human colonocytes to folate deficiency in vitro: functional and proteomic analysis. J. Proteome Res. 7 (2008) 3254-3266.

16. S.N. Wickramasinghe, S. Fida Bone marrow cells from vitamin $B_{12}$ - and folate-deficient patients misincorporate uracil into DNA. Blood 83 (1994) 1656-1661.

17. G.P. Basten, M.H. Hill, S.J. Duthie and H.J. Powers, Effect of folate supplementation on the folate status of buccal mucosa and lymphocytes. Cancer Epidemiol. Biomarkers and Prevention 13 (2004) 1244-1249.

18. A.P. McGlynn, G.R. Wasson, S.L. O.Reilly, H. McNulty, C.S. Downes, C-K. Chang, L. Hoey, A.M. Molloy, M. Ward, J.J. Strain, G. McKerr, D.G. Weir, J.M. Scott, Low colonocuyte folate is associated with uracil misincorporation and global DNA hypomethylation in human colorectum. J. Nutr. 143 (2013) 27-33.

19. S.L. O'Reilly, A.P. McGlynn, H. McNulty, J. Reynolds, G.R. Wasson, A.M. Molloy, J.J. Strain, D.G. Weir, M. Ward, G. McKerr, J.M. Scott, C.S. Downes, Folic Acid Supplementation in Postpolypectomy Patients in a Randomized Controlled Trial Increases Tissue Folate Concentrations and Reduces Aberrant DNA Biomarkers in Colonic Tissues Adjacent to the Former Polyp Site. J Nutr. 146 (2016) 933-939.

20. C.K. Kumar, M.P. Moyer, P.K. Dudja, H.M. Said, A Protein-tyrosine kinase-regulated, $\mathrm{pH}$-dependent, carrier-mediated uptake system for folate in human normal colonic epithelial cell Line NCM460. J. Biol. Chem. 272 (1997) 6226-6231. 
21. S. Matt, T.G. Hofmann, The DNA damage-induced cell death response: a roadmap to kill cancer cells. Cellular and Molecular Life Sciences 73 (2016) 28292850.

22. J. Little, L. Sharp, S. Duthie, S. Narayanan, Colon cancer and genetic variation in folate metabolism: the clinical bottom line. J. Nutr. 133 (2003) 3758S-3766S.

23. M. Kapiszewska, M. Kalembra, U. Wojciech, T. Milewicz, Uracil misincorporation into DNA of leukocytes of young women with positive folate balance depends on plasma vitamin B12 concentrations and methylenetetrahydrofolate reductase polymorphisms. A pilot study. J. Nutr. Biochem. 16 (2005) 467-478.

24. M. Rodríguez-Paredes, M. Esteller, Cancer epigenetics reaches mainstream oncology. Nat Med. 17 (2011) 330-339.

25. C. Gallois, P. Laurent-Puig, J. Taieb, Methylator phenotype in colorectal cancer: a prognostic factor or not? Crit. Rev. Oncol. Hematol. 99 (2016) 74-80.

26. J.F. Wentzel, C. Gouws, C. Huysamen et al. Assessing the DNA methylation status of single cells with the comet assay. Anal. Biochem. 400 (2010) 190-194.

27. A. Lewies, E. Van Dyk, J.F. Wentzel, P.J. Pretorius, Using a medium-throughput comet assay to evaluate the global DNA methylation status of single cells. Front. Genet. 5 (2014) article 215.

28. A. Perotti, V. Rossi, A. Mutti, A. Buschini, Methy-sens Comet assay and DNMTs transcriptional analysis as a combined approach in epigenotoxicology. Biomarkers 20 (2015) 64-70.

29. F. Kusari, A.M. O'Doherty, N.J. Hodges, M.W. Wojewodzic, Bi-directional effects of vitamin B12 and methotrexate on Daphnia magna fitness and genomic methylation. Sci. Rep. 7 (2017) article 11872.

30. A. Collins, A. Ma, S.J. Duthie, The kinetics of repair of oxidative DNA damage (strand breaks and oxidised pyrimidines) in human cells. Mutat. Res. 336 (1995) 69-77.

31. G.R. Wasson, A.P. McGlynn, H. McNulty, S.L. O'Reilly, V.J. McKelvey-Martin, G. McKerr, J.J. Strain, J. Scott, C.S. Downes, Global DNA and p53 region-specific hypomethylation in human colonic cells is induced by folate depletion and reversed by folate supplementation. J. Nutr. 136 (2006) 2748-2753. 